\title{
A pathoanatomic approach to secondary functional mitral regurgitation: Evaluating the evidence
} \author{
Gorav Ailawadi, MD
}

Vinay Badhwar, MD, ${ }^{\mathrm{a}}$ Mohamad Alkhouli, MD, ${ }^{\mathrm{b}}$ Michael J. Mack, MD, ${ }^{\mathrm{c}}$ Vinod H. Thourani, MD, ${ }^{\mathrm{d}}$ and

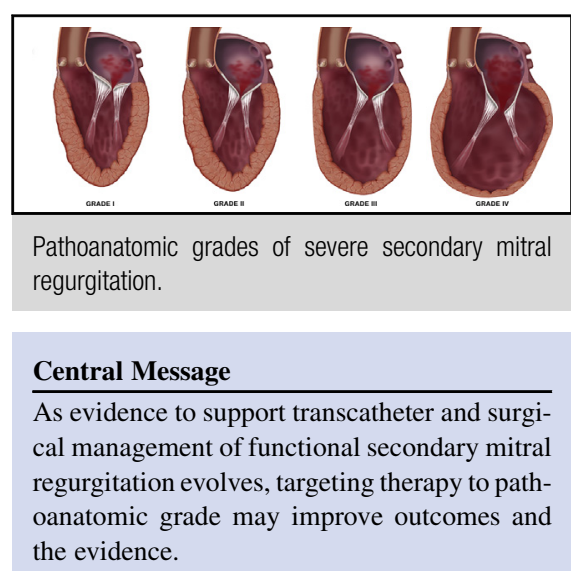

See Commentaries on pages 82 and 84 .
Leaflet tethering and annular dilatation secondary to left ventricular (LV) dilatation and papillary muscle displacement pose ongoing challenges to the surgical or transcatheter management of functional secondary mitral regurgitation (SMR). However, recent transcatheter trial results have put current treatment strategies into a new prospective.

The primary objective of this review is to summarize the existing evidence for transcatheter and surgical therapy in the context of recent Cardiovascular Outcomes Assessment of the MitraClip Percutaneous Therapy for Heart Failure Patients With Functional Mitral Regurgitation (COAPT) ${ }^{1}$ trial and Multicentre Study of Percutaneous Mitral Valve Repair MitraClip Device in Patients With Severe Secondary Mitral Regurgitation (MITRA-FR) ${ }^{2}$ results. The secondary objective is to begin to identify which patients may be best suited for transcatheter versus surgical therapy by proposing a simplified pathoanatomic schema for medical, transcatheter, and surgical approaches to SMR.

\section{Transcatheter Repair: Emerging New Role}

The lack of data demonstrating a survival benefit of corrective surgery for SMR, the higher mitral regurgitation (MR) recurrence rate following restrictive mitral valve (MV) annuloplasty ${ }^{3}$ and the higher mortality of MV replacement ${ }^{4}$ has prompted the search for alternative therapies for patients with SMR and LV dysfunction. Transcatheter mitral repair with the MitraClip device (Abbott, Santa Clara, Calif) has been studied as an alternative option to reduce MR and determine whether that reduction is associated with any clinical benefit in patients with heart failure (HF). ${ }^{5}$ The MitraClip is currently approved in the United States for the treatment of primary MR in those patients deemed to be prohibitive risk for surgical therapy. This regulatory approval is based on the Endovascular Valve Edgeto-Edge Repair Study (EVEREST) II investigational device exemption trial randomized MitraClip therapy to MV surgery for primary and secondary MR. ${ }^{6-8}$ Although the majority of MitraClip procedures in Europe are performed in patients with secondary MR, this has not followed suit in the United States due to lack of regulatory approval for this indication.

Only a small cohort of SMR patients were treated in EVEREST II; thus, the evidence of benefit with the MitraClip in SMR patients ${ }^{9}$ and is observational only and is summarized in a recent meta-analysis of global data. This meta-analysis has indicated that MitraClip may improve symptoms, reduce HF hospitalizations and possibly reduce cardiovascular mortality. ${ }^{10}$ However, the significant heterogeneity between the studies and the lack of adjudication of SMR severity and clinical outcomes precluded drawing definitive conclusions from these data. A recent subset analysis of 436 high-risk and 86 non-high-risk SMR patients from the EVEREST II trial and its continued access registry has revealed that if patients are discharged with $2+$ MR or less following MitraClip therapy, symptom improvement, LV remodeling, HF hospitalization reduction, and MR reduction can be maintained at 1 year. ${ }^{11}$ This large study begins to establish that there are some patients with SMR that may indeed benefit from edge-to-edge transcatheter therapy.

On the heels of this experience, 2 recent landmark prospective randomized clinical trials have been published studying the heterogeneous population of patients with SMR. The COAPT trial ${ }^{1}$ and the MITRA-FR ${ }^{2}$ both randomized SMR patients to receive the MitraClip + guidelinedirected medical therapy (GDMT) versus GDMT alone. 
Yet, whereas the same device was used to treat the same disease in 2 relatively large trials, quite different outcomes were observed.

\section{The COAPT Trial}

The COAPT trial randomized 614 patients with symptomatic HF and severe SMR (by US echocardiogram guideline criteria) 1:1 to receive GDMT + MitraClip versus GDMT alone. Patients with advanced ventricular dilation, those with LV ejection fraction $<20 \%$, and those with SMR less than moderate to severe were excluded. Patients had to be optimized and stable on maximal GDMT including resynchronization therapy for at least 3 months to be eligible for enrollment. The primary efficacy end point was all hospitalizations for HF within 24 months of followup. The primary safety end point was freedom from devicerelated complications at 12 months compared with a prespecified objective performance goal of $88 \%$. The trial dramatically met both of its primary efficacy and safety end points; the annualized rate of all HF hospitalizations within 24 months was $35.8 \%$ per patient-year in the device group compared with $67.9 \%$ per patient-year in the control group (hazard ratio [HR], 0.53; 95\% confidence interval [CI], $0.40-0.70 ; P<.001$; number needed to treat $=3$ ). The rate of freedom from device-related complications at 12 months was $96.6 \%$ (lower $95 \%$ confidence limit, $94.8 \% ; P<.001$ for comparison with the performance goal). In addition, patients in the MitraClip group had a substantial reduction in all-cause mortality at 24 months (29.1\% vs $46.1 \%$; HR, 0.62; 95\% CI, 0.46-0.82; $P<$ .001 ; number needed to treat $=6$ ). Also of note, the intervention group did significantly better than the GDMT alone group in all 10 of the prespecified hierarchical secondary end points, including objective and patient reported quality of life measures, and echocardiographic remodeling parameters.

These impressive results are expected to influence future guidelines and receive regulatory approval for the use of MitraClip for specific patients with SMR. This rigorously performed study has brought evidence of benefit for many sick HF patients in whom uncertainties about a beneficial influence of SMR elimination have precluded them from referrals for correction of their SMR. Despite these exciting findings, concerns have arisen regarding the generalizability of these results to the highly heterogeneous groups of SMR patients. This is particularly due to the optimization of GDMT and the strict inclusion and exclusion criteria that were applied in the trial leading to the actual enrollment of only $42 \%$ of the screened cohort, without data on this screen-fail group being readily available. Furthermore, an important exclusion criterion was that patients were deemed not to be candidates for surgery as offered by the institutional heart teams. Although the widespread potential of applicability to all SMR patients with HF is indeed plausible, to avoid potential overinterpretation of these results some equipoise may be warranted as a broader pool of evidence is examined. The question remains how generalizable are the results of this trial to the real world?

\section{MITRA-FR}

In contrast to the COAPT trial, MITRA-FR permitted patients with more advanced degrees of LV dilation and less severe SMR to be enrolled in their study design. Also, GDMT both at baseline and during follow-up was determined locally and did not require a strict central eligibility committee adjudication and was not monitored during the trial. The trial randomized 304 patients $(72 \%$ of screened patients) with symptomatic HF and severe SMR (by European criteria) 1:1 to receive GDMT + MitraClip versus GMDT alone. The primary efficacy end point was a composite of death from any cause or unplanned hospitalization for $\mathrm{HF}$ at 12 months (vs 24 months in COAPT). At the conclusion of the study there was no difference in the rate of the primary outcome between the intervention group and the control group $(54.6 \%$ vs $51.3 \%$; odds ratio, 1.16 ; $95 \%$ CI, 0.73-1.84). There were also no differences in the rates of all-cause mortality death $(24.3 \%$ vs $22.4 \%$; HR, $1.11 ; 95 \%$ CI, $0.69-1.77)$ or unplanned HF hospitalizations (48.7\% vs $47.4 \%$; HR, 1.13 ; $95 \%$ CI, $0.81-1.56$ ). The study concluded that among patients with severe SMR, the rate of death or unplanned hospitalization for HF at 1 year did not differ significantly between patients who underwent transcatheter MV repair with the MitraClip device in addition to receiving GDMT and those who received GDMT alone.

So why did the COAPT trial achieve its study end points, whereas MITRA-FR showed no benefit? It appears that whereas both trials examined MitraClip + GDMT versus GDMT alone, the 2 trials may have examined clinically and pathoanatomically slightly different populations (Table 1). It is recognized that GDMT may provide benefit to symptoms and ventricular remodeling and it should be part of the structural management of SMR. The COAPT trial enrolled patients who were optimized on maximal GDMT with moderately dilated ventricles, and perhaps less severe leaflet tethering. MITRA-FR took what might be interpreted as a more pragmatic realworld approach by allowing patients with less-optimized GDMT and more dilated ventricles, thus exposing the potential for more severe MV leaflet tethering. In addition, in the COAPT trial, there was greater severity of MR with twice the length of follow-up. These differences indicate that the benefit of MitraClip may extend beyond 1 year and that patients with the worst ventricles, as defined by ejection fraction and LV volumes, and moderate degrees of MR were "nonresponders" in the COAPT trial receiving no benefit. 
TABLE 1. Comparison between Multicentre Study of Percutaneous Mitral Valve Repair MitraClip Device in Patients With Severe Secondary Mitral Regurgitation (MITRA-FR) and Cardiovascular Outcomes Assessment of the MitraClip Percutaneous Therapy for Heart Failure Patients With Functional Mitral Regurgitation (COAPT) Trial

\begin{tabular}{lcc}
\hline \multicolumn{1}{c}{ Variable } & MITRA-FR $(\mathbf{N}=\mathbf{3 0 4})$ & COAPT $(\mathbf{N}=\mathbf{6 1 4})$ \\
\hline Eligibility criteria & & $20-50$ \\
LVEF range $(\%)$ & $15-40$ & II, III, ambulatory IV \\
NYHA functional class & II, III, IV & EROA $>30 \mathrm{~mm}{ }^{2}, \mathrm{RV}>45 \mathrm{~mL} / \mathrm{beat}$ \\
SMR severity & EROA $>20 \mathrm{~mm}^{2}, \mathrm{RV}>30 \mathrm{~mL} / \mathrm{beat}$ & $41 \pm 15$ \\
EROA mean $\left(\mathrm{mm}^{2}\right)$ & $31 \pm 10 \mathrm{LVEDV}$ & $101 \pm 34$ \\
LVEDV mean $\left(\mathrm{mL}^{2} \mathrm{~m}^{2}\right)$ & $135 \pm 35$ & $\leq 70 \mathrm{~mm}$ \\
LVEDD & No requirements & Determined by CEC \\
GDMT at baseline & Determined by local PIs & Adjustments required justification and \\
GDMT during follow-up & Adjustments allowed but were not & were thoroughly documented \\
Follow-up duration $(\mathrm{mo})$ & collected & 24 \\
Acute outcomes & 12 & 5.5 \\
Acute results: no clip $/ \geq 3+$ MR $(\%)$ & 9 & 5 \\
Periprocedural complications $(\%)$ & 14.6 & 5 \\
MR $<+3$ at 12 mo $(\%)$ & 17 &
\end{tabular}

Long-term outcomes

Primary efficacy end points

Death or HF hospitalizations at $12 \mathrm{mo}$

All HF hospitalizations at $24 \mathrm{mo}$

$54.6 \%$ vs $51.3 \%(P=.53)$

$35.8 \%$ vs $67.9 \%$ PPY $(P<.001)$

$L V E F$, Left ventricular ejection fraction; $N Y H A$, New York Heart Association; $S M R$, functional secondary mitral regurgitation; $E R O A$, effective regurgitant orifice area; $R V$, regurgitant volume; $L V E D V$, left ventricular end diastolic volume; $L V E D D$, left ventricular end diastolic diameter; GDMT, guideline directed medical therapy; $P I$, principal investigator; $C E C$, central eligibility committee; $M R$, mitral regurgitation; $H F$, heart failure; $P P Y$, patient per year.

The striking differences in the outcomes of these 2 trials highlight a key fact: SMR is not a 1-size-fits-all entity.

\section{Surgical Therapy: Still Relevant?}

Despite the prevailing impressions from recent randomized evidence in ischemic SMR, suggesting that patients requiring surgery should be considered for MV replacement instead of repair for improved durability, ${ }^{12,13}$ the most common surgical approach to SMR in the United States is still MV repair, predominantly as a concomitant operation. ${ }^{4,14}$ Clinical registry data revealed that in 2016, 2885 patients underwent coronary artery bypass grafting and MV replacement with a 9.5\% 30-day operative mortality rate, and 3464 patients underwent coronary artery bypass grafting with concomitant MV repair with a $4.6 \%$ mortality. ${ }^{4}$ Although these procedure-related data serve only as a surrogate to SMR etiology, when etiology was specifically examined to determine its influence on MV operation outcome, SMR was found not to be an independent predictor of 30-day mortality or major morbidity. ${ }^{15}$ This indicates that outcomes of patients undergoing surgery for SMR are likely dictated more by a patient's comorbidity profile and operative strategy rather than etiology itself. However, these data do not examine the longitudinal influence of MV repair versus MV replacement. The 2-year results of the Cardiothoracic Surgical Trials Network examination between repair and replacement in SMR helps to make 2 important points for our evidence-based interpretation of the midterm outcome of SMR operations. ${ }^{13}$ First,
MV replacement as an operative strategy clearly has better longitudinal durability and affords improved reduction of reoperations for recurrent regurgitation. Second, there is a near doubling of upfront mortality risk compared with MV repair that lasts through follow-up, similar to the US clinical registry data. ${ }^{4}$ Although overall mortality was not significantly different between the 2 groups, patients with a durable MV repair had the greatest degree of LV remodeling and survival.

These findings from the recent surgical literature, also supported by the transcatheter literature, highlight a key fact: SMR is not a 1-size-fits-all entity.

This should not come as a surprise to surgeons. Efforts to determine why some patients do very well with MV repair for SMR but some fare poorly has been the focus of investigation for years. ${ }^{16-20}$ A pathologic spectrum in SMR exists that involves an interplay of the degree of ventricular dilatation and function, leaflet tethering, annular dilatation, and loading conditions. Drake and colleagues, ${ }^{21}$ assimilated preoperative echocardiographic predictors into clinical decision making to tailor the surgical approach and increase successful repair rates for SMR in real-world practice. Kron and colleagues ${ }^{22}$ examined the repair failures of the Cardiothoracic Surgical Trials Network experience and identified that severe posterior leaflet tethering and inferobasal ventricular aneurysm formation predicted long-term failure of MV repair. The American Association for Thoracic Surgery summarized these findings in 2016 with recommendations that MV 
replacement rather than repair should be considered in these patients and in those with LV end diastolic dimensions $>65 \mathrm{~mm}^{23}$ To further explore how the subvalvular apparatus may influence outcome of MV repair, several investigators have shown favorable outcomes with papillary muscle remodeling procedures. Application of a polytetrafluoroethylene tube or sling at the base of the papillary muscles for active reapproximation may reduce leaflet tethering and improve outcomes of restrictive annuloplasty. ${ }^{24} \mathrm{~A}$ suture between the posteromedial papillary muscle and the posterior annulus has also been shown to improve repair outcomes longitudinally. ${ }^{25}$ These maneuvers, once thought to be isolated to few centers and not reproducible, are becoming more commonly applied with simplified techniques and providing very encouraging outcomes. ${ }^{26-29}$ Finally, as we interpret the success of MitraClip with certain pathoanatomic presentations, it may be of relevance to note that the longitudinal surgical success of the edge-to-edge technique popularized by De Bonis and colleagues $^{30}$ occurs only when an annuloplasty is added.

\section{Transcatheter MV Replacement: Where to Next?}

Because the recent evidence involving transcatheter MV repair, ${ }^{1}$ transcatheter $\mathrm{MV}$ replacement held the hopes of many as a potential answer to HF patients with SMR that were of high risk for conventional surgical MV replacement. $^{27}$ The 2 most-developed technologies are the Intrepid valve (Medtronic Inc, Minneapolis, Minn) and the Tendyne valve (Abbott, Santa Clara, Calif), both demonstrating quite encouraging early outcomes and both in active US pivotal trial investigation. ${ }^{31-34}$

What is to become of current trials given the results of the COAPT trial? This remains a topic of some controversy because the collective evidence is weighed by the scientific community, guideline committees, as well as the Food and Drug Administration and the Centers for Medicare and Medicaid Services. Because there remains some equipoise in the evidence, and there is general consensus that not all SMR patients are ideal candidates for a single transcatheter or surgical therapy or perhaps any intervention at all, there is likely room for all options as they find their optimal therapeutic indication.

\section{A Pathoanatomic Approach to Future Management}

Surgical repair indeed has its niche role in pathoanatomically suitable disease 21,23,27,29 and MV replacement has its place for symptom resolution, although randomized investigation has not been performed to clearly identify survival benefit. ${ }^{12,13}$ Transcatheter repair will clearly have an expanding role in SMR, but successes in some patients and failures in others highlight the need for clarity in how to identify the ideal patients to receive optimal benefit from this modality. ${ }^{1,2,8-10}$ Transcatheter MV replacement is undergoing active investigation, but anatomic limitations of LV outflow tract obstruction and annular size may limit its widespread application. Due to the spectrum of structural cardiac disease that accompanies SMR, not all patients can be managed in the same manner and therapy should be tailored.

In light of the accumulated knowledge from the surgical and transcatheter literature, a simplified pathoanatomic classification scheme of SMR that incorporates the key categories of annular dilatation, LV dysfunction/dilatation and leaflet tethering is proposed (Figure 1). This scoring system may aid in assessing SMR features in outpatient clinics and echocardiography laboratories (Table 2) and assist in

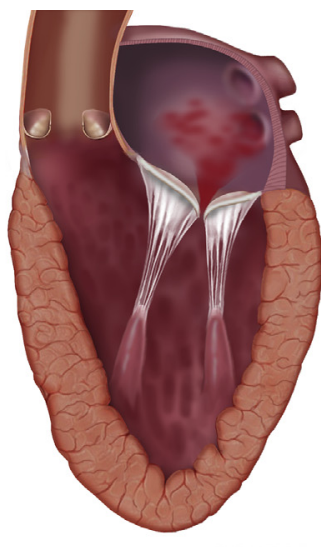

GRADE I

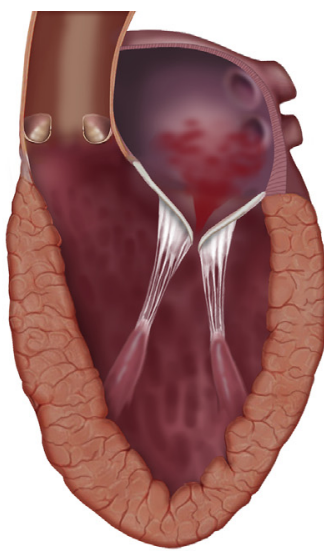

GRADE II

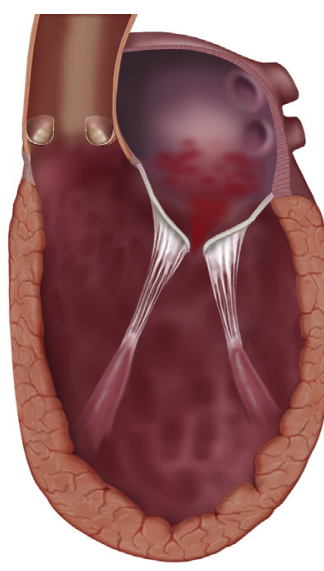

GRADE III

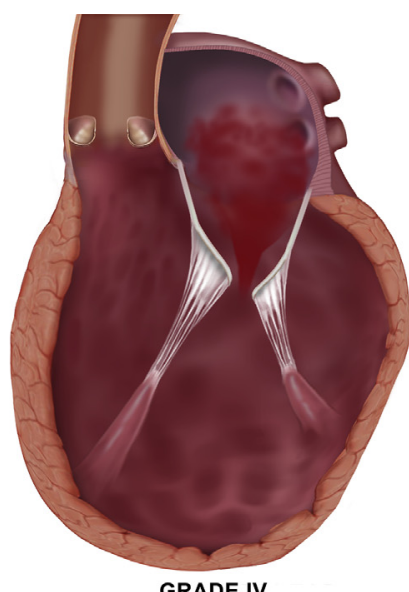

GRADE IV

FIGURE 1. Pathoanatomic grades of severe secondary mitral regurgitation. Grade I = Mild-moderate annular dilation, ejection fraction $>45 \%$, minimal leaflet tethering $(<5 \mathrm{~mm})$. Grade II = Moderate-severe annular dilation, ejection fraction $35 \%$ to $45 \%$, moderate leaflet tethering $(5-10$ mm). Grade $\mathrm{III}=$ Severe annular dilation, ejection fraction $25 \%$ to $35 \%$, severe leaflet tethering ( $>10 \mathrm{~mm})$. Grade IV = Severe annular dilation, ejection fraction $<25 \%$ or inferobasilar dysfunction, severe leaflet tethering $(>10 \mathrm{~mm})$. 
TABLE 2. Pathoanatomic classification of secondary mitral regurgitation

\begin{tabular}{llcc}
\hline Grade & \multicolumn{1}{c}{ Annular dilatation } & Ejection fraction $(\%)$ & Leaflet tethering \\
\hline 1 & Mild to moderate $(3-4 \mathrm{~cm})$ & $>45$ & Mild $(<5 \mathrm{~mm})$ \\
2 & Moderate to severe $(4-5 \mathrm{~cm})$ & $35-45$ & Moderate $(5-10 \mathrm{~mm})$ \\
3 & Severe $(>5 \mathrm{~cm})$ & $25-35$ & Severe $(>10 \mathrm{~mm})$ \\
4 & Severe $(>5 \mathrm{~cm})$ & $<25$ or presence of inferobasal dysfunction & Severe $(>10 \mathrm{~mm})$ \\
\hline
\end{tabular}

tailoring the pathoanatomy to possible therapeutic options based on comorbid risk (Table 3 ). This intuitive schema may be useful for future prospective investigation as well as for retrospective examination of both the MITRA-FR echocardiograms as well as the COAPT trial core lab echocardiograms to ascertain if this simple categorization predicts short- and long-term success in transcatheter MV repair.

\section{Conflict of Interest Statement}

Dr Badhwar is a consultant for Abbott and serves as the global program committee co-chair for Tendyne (uncompensated), Dr Mack is a consultant for Medtronic and Abbott and serves as the executive committee co-chair for the Transcatheter Mitral Valve Replacement With the Medtronic Intrepid TMVR System in Patients With Severe Symptomatic Mitral Regurgitation (APOLLO) trial and

TABLE 3. Pathoanatomic management of secondary mitral regurgitation

Grade 1

GDMT and surgical MV repair in setting of concomitant operations in low- to moderate-risk patients

GDMT and TMVr for patients deemed not suitable for surgery by the heart team

Grade 2

GDMT and surgical MV repair or MVR

GDMT and TMVr for patients deemed not suitable for surgery by the heart team

GDMT and TMVR (within clinical trial) in high-risk patients deemed not suitable for surgery or $\mathrm{TMVr}$

Grade 3

GDMT and surgical MVR in low- to moderate-risk patients

GDMT and TMVR (within clinical trial) in high-risk patients deemed Grade 4

$$
\text { not suitable for surgery }
$$

GDMT and advanced HF therapy (VAD/OHTx or medical palliation)

\begin{tabular}{lll}
\hline Grade & Low risk & High risk \\
\hline 1 & MVr \pm CABG & TMVr \\
2 & MVR \pm CABG & TMVr or TMVR \\
3 & & TMVR \\
4 & & \\
\hline
\end{tabular}

GDMT, Guideline-directed medical therapy; $M V$, mitral valve; $T M V r$, transcatheter mitral valve repair; $M V R$, mitral valve replacement; $T M V R$, transcatheter mitral valve replacement; $H F$, heart failure; $V A D$, ventricular assist device; $O H T x$, orthotopic heart transplantation; $M V r$, mitral valve repair; $C A B G$, coronary artery bypass grafting. co-principle investigator of the Cardiovascular Outcomes Assessment of the MitraClip Percutaneous Therapy for Heart Failure Patients With Functional Mitral Regurgitation (COAPT) trial (expenses only). Dr Thourani is a consultant for Edwards and Abbott and serves as the co-principle investigator for the Tendyne mitral annular calcification trial. Dr Ailawadi is a consultant for Abbott and serves as the co-principle investigator for the Tendyne Clinical Trial to Evaluate the Safety and Effectiveness of Using the Tendyne Mitral Valve System for the Treatment of Symptomatic Mitral Regurgitation (SUMMIT) trial. Dr Alkhouli has nothing to disclose with regard to commercial support.

\section{References}

1. Stone GW, Lindenfeld J, Abraham WT, Kar S, Lim DS, Mishell JM, et al. Transcatheter mitral-valve repair in patients with heart failure. N Engl J Med. 2018; 379:2307-18.

2. Obadia JF, Messika-Zeitoun D, Leurent G, Iung B, Bonnet G, Piriou N, et al. Percutaneous repair or medical treatment for secondary mitral regurgitation. $N$ Engl J Med. 2018;379:2297-306.

3. Wu AH, Aaronson KD, Bolling SF, Pagani FD, Welch K, Koelling TM. Impact of mitral valve annuloplasty on mortality risk in patients with mitral regurgitation and left ventricular systolic dysfunction. J Am Coll Cardiol. 2005;45:381-7.

4. D’Agostino RS, Jacobs JP, Badhwar V, Fernandez FG, Paone G, Wormuth DW, et al. The Society of Thoracic Surgeons adult cardiac surgery database: 2018 update on outcomes and quality. Ann Thorac Surg. 2018;105:15-23.

5. Goel SS, Bajaj N, Aggarwal B, Gupta S, Poddar KL, Ige M, et al. Prevalence and outcomes of unoperated patients with severe symptomatic mitral regurgitation and heart failure: comprehensive analysis to determine the potential role of MitraClip for this unmet need. J Am Coll Cardiol. 2014;63:185-6.

6. Mauri L, Garg P, Massaro JM, Foster E, Glower D, Mehoudar P, et al. The EVEREST II trial: design and rationale for a randomized study of the Evalve MitraClip System compared with mitral valve surgery for mitral regurgitation. Am Heart J. 2010;160:23-9.

7. Glower DD, Kar S, Trento A, Lim DS, Bajwa T, Quesada R, et al. Percutaneous mitral valve repair for mitral regurgitation in high-risk patients: results of the EVEREST II study. J Am Coll Cardiol. 2014;64:172-81.

8. Feldman T, Kar S, Elmariah S, Smart SC, Trento A, Siegel RJ, et al. Randomized comparison of percutaneous repair and surgery for mitral regurgitation: 5-year results of EVEREST II. J Am Coll Cardiol. 2015;66:2844-54.

9. Sorajja P, Mack M, Vemulapalli S, Holmes DR, Stebbins A, Kar S, et al. Outcomes of the initial experience with commercial transcatheter mitral valve repair in the United States. J Am Coll Cardiol. 2016;67:1129-40.

10. De Rosa R, Silverio A, Baldi C, Di Maio M, Prota C, Radano I, et al. Transcatheter repair of functional mitral regurgitation in heart failure patients: a metaanalysis of 23 studies on MitraClip implantation. Circ J. 2018;82:2800-10.

11. Ailawadi G, Lim DS, Mack MJ, Trento A, Kar S, Grayburn PA, et al. One year outcomes following MitraClip for functional mitral regurgitation. Circulation. 2018;139:37-47.

12. Acker MA, Parides MK, Perrault LP, Moskowitz AJ, Gelijns AC, Voisine P, et al. Mitral-valve repair versus replacement for severe ischemic mitral regurgitation. N Engl J Med. 2014;370:23-32.

13. Goldstein D, Moskowitz AJ, Gelijns AC, Ailawadi G, Parides MK, Perrault LP, et al. Two-year outcomes of surgical treatment of severe ischemic mitral regurgitation. N Engl J Med. 2016;374:344-53. 
14. Gammie JS, Chikwe J, Badhwar V, Thibault DP, Vemulapalli S, Thourani VH, et al. Isolated mitral valve surgery: the Society of Thoracic Surgeons adult cardiac surgery database analysis. Ann Thorac Surg. 2018;106:716-27.

15. Rankin JS, Grau-Sepulveda M, Shahian DM, Gillinov AM, Suri R, Gammie JS, et al. The impact of mitral disease etiology on operative mortality after mitral valve operations. Ann Thorac Surg. 2018;106:1406-13.

16. Haan CK, Cabral CI, Conetta DA, Coombs LP, Edwards FH. Selecting patients with mitral regurgitation and left ventricular dysfunction for isolated mitral valve surgery. Ann Thorac Surg. 2004;78:820-5.

17. Magne J, Pibarot P, Dumesnil JG, Senechal M. Continued global left ventricular remodeling is not the sole mechanism responsible for the late recurrence of ischemic mitral regurgitation after restrictive annuloplasty. J Am Soc Echocardiogr. 2009;22:1256-64.

18. Lee AP, Acker M, Kubo SH, Bolling SF, Park SW, Bruce CJ, et al. Mechanisms of recurrent functional mitral regurgitation after mitral valve repair in nonischemic dilated cardiomyopathy: importance of distal anterior leaflet tethering. Circulation. 2009;119:2606-14.

19. Crabtree TD, Bailey MS, Moon MR, Munfakh N, Pasque MK, Lawton JS, et al. Recurrent mitral regurgitation and risk factors for early and late mortality after mitral valve repair for functional ischemic mitral regurgitation. Ann Thorac Surg. 2008;85:1537-42

20. Onorati F, Rubino AS, Marturano D, Pasceri E, Santarpino G, Zinzi S, et al. Midterm clinical and echocardiographic results and predictors of mitral regurgitation recurrence following restrictive annuloplasty for ischemic cardiomyopathy. J Thorac Cardiovasc Surg. 2009;138:654-62.

21. Drake DH, Zimmerman KG, Hepner AM, Nichols CD. Echo-guided mitral repair. Circ Cardiovasc Imaging. 2014;7:132-41.

22. Kron IL, Hung J, Overbey JR, Bouchard D, Gelijns AC, Moskowitz AJ, et al. Predicting recurrent mitral regurgitation after mitral valve repair for severe ischemic mitral regurgitation. J Thorac Cardiovasc Surg. 2015;149:752-61.

23. Kron IL, Acker MA, Adams DH, Ailawadi G, Bolling SF, Hung JW, et al. 2015 the American Association for Thoracic Surgery consensus guidelines: ischemic mitral valve regurgitation. J Thorac Cardiovasc Surg. 2016;151:940-56.
24. Hvass U, Joudinaud T. The papillary muscle sling for ischemic mitral regurgitation. J Thorac Cardiovasc Surg. 2010;139:418-23.

25. Fattouch K, Castrovinci S, Murana G, Dioguardi P, Guccione F, Nasso G, et al Papillary muscle relocation and mitral annuloplasty in ischemic mitral valve regurgitation: midterm results. J Thorac Cardiovasc Surg. 2014;148:1947-50.

26. Harmel EK, Reichenspurner H, Girdauskas E. Subannular reconstruction in sec ondary mitral regurgitation: a meta-analysis. Heart. 2018;104:1783-90.

27. Kainuma S, Funatsu T, Kondoh H, Yokota T, Maeda S, Shudo Y, et al. Beneficial effects of restrictive annuloplasty on subvalvular geometry in patients with functional mitral regurgitation and advanced cardiomyopathy. J Thorac Cardiovasc Surg. 2018;156:630-8.

28. Misumi Y, Masai T, Toda K, Nakamura T, Miyagawa S, Yoshikawa Y, et al Restrictive mitral annuloplasty with or without papillary muscle approximation for functional mitral regurgitation. J Heart Valve Dis. 2017;26:447-55.

29. Nappi F, Spadaccio C, Nenna A, Lusini M, Fraldi M, Acar C, et al. Is subvalvular repair worthwhile in severe ischemic mitral regurgitation? Subanalysis of the papillary muscle approximation trial. J Thorac Cardiovasc Surg. 2017;153: 286-95.

30. De Bonis M, Lapenna E, Pozzoli A, Giacomini A, Alfieri O. Edge-to-edge surgical mitral valve repair in the era of MitraClip: what if the annuloplasty ring is missed? Curr Opin Cardiol. 2015;30:155-60.

31. Badhwar V, Thourani VH, Ailawadi G, Mack MJ. Transcatheter mitral valve therapy: the event horizon. J Thorac Cardiovasc Surg. 2016;152: 330-6.

32. Bapat V, Rajagopal V, Meduri C, Farivar RS, Walton A, Duffy SJ, et al. Early experience with new transcatheter mitral valve replacement. J Am Coll Cardiol. 2018;71:12-21.

33. Meredith I, Bapat V, Morriss J, McLean M, Prendergast B. Intrepid transcatheter mitral valve replacement system: technical and product description. EuroIntervention. 2016;12:Y78-80.

34. Muller DWM, Farivar RS, Jansz P, Bae R, Walters D, Clarke A, et al. Transcatheter mitral valve replacement for patients with symptomatic mitral regurgitation: a global feasibility trial. J Am Coll Cardiol. 2017;69:381-91. 